Background: Apolipoprotein E (APOE) $\epsilon 4$ allele is a very well-known genetic risk factor of Alzheimer's disease (AD). Recent histopatholgical and MRI studies in patients with AD demonstrated alterations of white matter, as well as gray matter. This study aimed to investigate possible associations between cerebral white matter changes and APOE genotype in early stage AD. Methods: The authors used Diffusion Tensor Imaging (DTI) to explore cerebral white matter changes to detect pathological processes. $26 \mathrm{AD}$ participants were included in the study of which 13 were APOE $\epsilon 4$ carriers and age-, gender-, and severity-matched 13 were APOE $\epsilon 4$ non-carriers. All AD individuals were met for NINCDS-ADRDA Diagnostic Criteria of probable AD and their global scores of Clinical Dementia Rating scale were 0.5 or 1 . Voxel-based comparisons for the Fractional Anisotropy (FA) and Mean Diffusivity (MD) were performed between APOE $\epsilon 4$ carriers and $\epsilon 4$ non-carriers using SPM5. Results: FA value was significantly lower in APOE $\epsilon 4$ carriers than APOE $\epsilon 4$ non-carriers in the bilateral temporal white matter, right cingulate white matter, left parahippocampal white matter, right postcentral parietal white matter, bilateral frontal and occipital white matter. Compared to APOE $\epsilon 4$ non-carriers, APOE $\epsilon 4$ carriers had significant increase of the MD value in the bilateral medial frontal white matter, right inferior frontal white matter, left precentral white matter, and left posterior cingulate white matter $(\mathrm{p}<0.005$, uncorrected for multiple comparisons). Conclusions: A1though the exact mechanism underlying vulnerability of white matter tracts in APOE $\epsilon 4$ carriers is still unclear, these findings indicate that increased genetic risk by APOE $\epsilon 4$ allele is associated with advanced regional alterations of white matter, as well as gray matter changes, in early stage $\mathrm{AD}$.

\section{P2-037 CATEGORICAL AND CORRELATIONAL ANALYSES OF BASELINE FLUORODEOXYGLUCOSE POSITRON EMISSION TOMOGRAPHY IMAGES FROM THE ALZHEIMER'S DISEASE NEUROIMAGING INITIATIVE}

Wendy Lee ${ }^{1,2}$, Jessica B. S. Langbaum ${ }^{1,2}$, Kewei Chen ${ }^{1,2}$, Cole Recshke ${ }^{1,2}$, Daniel Bandy ${ }^{1,2}$, Gene E. Alexander ${ }^{3,2}$, Norman L. Foster ${ }^{4}$, Michael W. Weiner ${ }^{5}$, Robert A. Koeppe ${ }^{6}$, William J. Jagust ${ }^{7}$, Eric M. Reiman ${ }^{1,2},{ }^{1}$ Banner Alzheimer's Institute, Phoenix, AZ, USA ${ }^{2}$ Arizona Alzheimer's Consortium, Phoenix, AZ, USA; ${ }^{3}$ University of Arizona, Tucson, AZ, USA; ${ }^{4}$ University of Utah, Salt Lake City, UT, USA; ${ }^{5}$ University of California, San Francisco, CA, USA; ${ }^{6}$ University of Michigan, Ann Arbor, MI, USA; ${ }^{7}$ University of California Davis, Davis, CA, USA. Contact e-mail:

Wendy.Lee@bannerhealth.com

Background: Alzheimer's disease (AD) is characterized by regional reductions in fluorodeoxyglucose positron emission tomography (PET) measurements of the cerebral metabolic rate for glucose (CMRgl), which are correlated with clinical severity, progressive and apparent before the onset of dementia or mild cognitive impairment (MCI). We have been using statistical parametric mapping (SPM) to analyze PET data from probable AD patients, amnestic MCI (aMCI) patients and normal controls (NC) from the AD Neuroimaging Initiative (ADNI). Our objective was to characterize patterns of cerebral hypometabolism in probable $\mathrm{AD}$ and $\mathrm{aMCI}$, and correlate lower Mini-Mental State Examination (MMSE) scores with lower regional CMRgl in both the overall cohort of probable $\mathrm{AD}, \mathrm{aMCI}$ and $\mathrm{NC}$ subjects and the probable AD group. Methods: Baseline PET images from 298 subjects, including 74 probable $\mathrm{AD}$ (age $70 \pm 10$, MMSE 23.5 \pm 2.2 ), 142 aMCI (age $67 \pm 12$, MMSE $27.1 \pm 1.7)$ and $82 \mathrm{NC}(69 \pm 10$ years old, MMSE 28.9 \pm 1.1 ), were analyzed on a voxel-by-voxel basis using SPM5. ANOVA with pair-wise comparisons contrasted regional CMRgl in the three subject groups. Linear regression correlated lower MMSE scores with lower CMRgl in the overall cohort and probable AD group. Results: Compared to NC, the probable AD and aMCI groups each had lower CMRgl bilaterally in previously characterized posterior cingulate, precuneus, and parietotemporal regions $(\mathrm{P}<0.05$ corrected for multiple comparisons), and occipital cortex, hippocampus, parahippocampal gyrus and fusiform gyrus (uncorrected $\mathrm{P}<0.005$ ). Compared to $\mathrm{NC}$, the probable $\mathrm{AD}$ group had lower $\mathrm{CMRgl}$ in frontal regions (corrected $\mathrm{P}<0.05$ ). In the overall cohort, lower MMSE scores were correlated with lower CMRgl in the posterior cingulate, precuneus, parietotemporal and frontal regions $(\mathrm{P}<0.05$, corrected for multiple comparisons). In the probable AD group, lower MMSE scores were correlated with lower $\mathrm{CMRgl}$ in left frontal and temporal regions (uncorrected $\mathrm{P}<0.001$ ). Conclusions: Findings from this large multicenter study confirm the previously characterized pattern of regional hypometabolism in $\mathrm{AD}$ and aMCI and implicate additional brain regions. Furthermore, they confirm the previously characterized correlation between severity of clinical impairment in a combined group of patients and controls, but suggest strongest correlations between clinical severity of frontal and temporal regions by the time AD patients satisfy criteria for dementia.

\section{P2-038 ATROPHY PATTERNS IN ALZHEIMER'S DISEASE AND SEMANTIC DEMENTIA: A COMPARISON OF FREESURFER AND MANUAL MEASUREMENTS}

Manja Lehmann ${ }^{1}$, Abdel Douiri ${ }^{1}$, Josephine Barnes ${ }^{1}$, Dennis Chan ${ }^{1}$, Martin N. Rossor ${ }^{1,2}$, Nick C. Fox ${ }^{1},{ }^{1}$ Dementia Research Centre, London, United Kingdom; ${ }^{2}$ Faculty of Medicine, Imperial College of Science, Engineering and Medicine, London, United Kingdom. Contact e-mail: lehmann@drc.ion.ucl.ac.uk

Background: Delineation of temporal lobe structures from MRI scans is increasingly used in research in dementia. The gold standard of manual delineation is time-consuming and subject to operator error. FreeSurfer is a freely-available automated technique which allows quantification of cortical thickness and a facility to label brain regions automatically. Automated regional labelling may be particularly difficult in cases with focal or generalised atrophy. We wished to compare FreeSurfer regions with manual delineation in such cases. Methods: MRI scans of 10 controls, 10 Alzheimer's disease (AD), and 10 semantic dementia (SD) subjects were included. SD is a clinical subtype of fronto-temporal dementia where temporal lobe gyri may be severely and focally atrophic. Temporal lobe regions were segmented by FreeSurfer and compared with manually-delineated regions. Results: Absolute volumes from FreeSurfer differed from manual volumes to different extents depending on region and subject group but were largely due to differences in protocols - with more temporal stem white matter included in FreeSurfer regions. Volume differences (FreeSurfer-manual) were different between controls and SD $(\mathrm{p}<0.05)$, but not between control-AD or between AD-SD groups. Importantly however, expressed as fraction of control volume the FreeSurfer results showed a very similar pattern of loss to that found with manual measures (Table). In the SD group the most severely atrophied regions were the left entorhinal cortex, left amygdala and left parahippocampal gyrus. Furthermore, greatest atrophy in the AD group was shown bilaterally in the entorhinal cortex and hippocampus. There was no evidence for leftright asymmetry in the control or AD groups. In the SD group the left temporal volumes were significantly smaller than the equivalent rightsided structures $(\mathrm{p}<0.0001)$ (Figure). Conclusions: Despite absolute volumes differing for FreeSurfer and manual segmentations, betweengroup comparisons revealed remarkably similar patterns of loss with the two methods: in $\mathrm{AD}$ symmetrical losses were most marked in the entorhinal cortex and hippocampus whereas SD showed profound asymmetrical, predominantly left-sided, atrophy affecting in particular the anterior and medial temporal lobe structures. 